\title{
Norois
}

Environnement, aménagement, société

\section{Fonctionnement géomorphologique des régions nord sahéliennes : exemple des cordons d'Ajar dans l'Ouest du Hodh mauritanien}

Geomorphological processes in the north sahelian regions: the example of the Ajar aeolian sandy system in the West mauritanian Hodh

Marie-Josée Penven , Adeline Cotonnec, Mohamed Lemine Ould El Hacen et Ahmed Ould Diah

\section{OpenEdition \\ Journals}

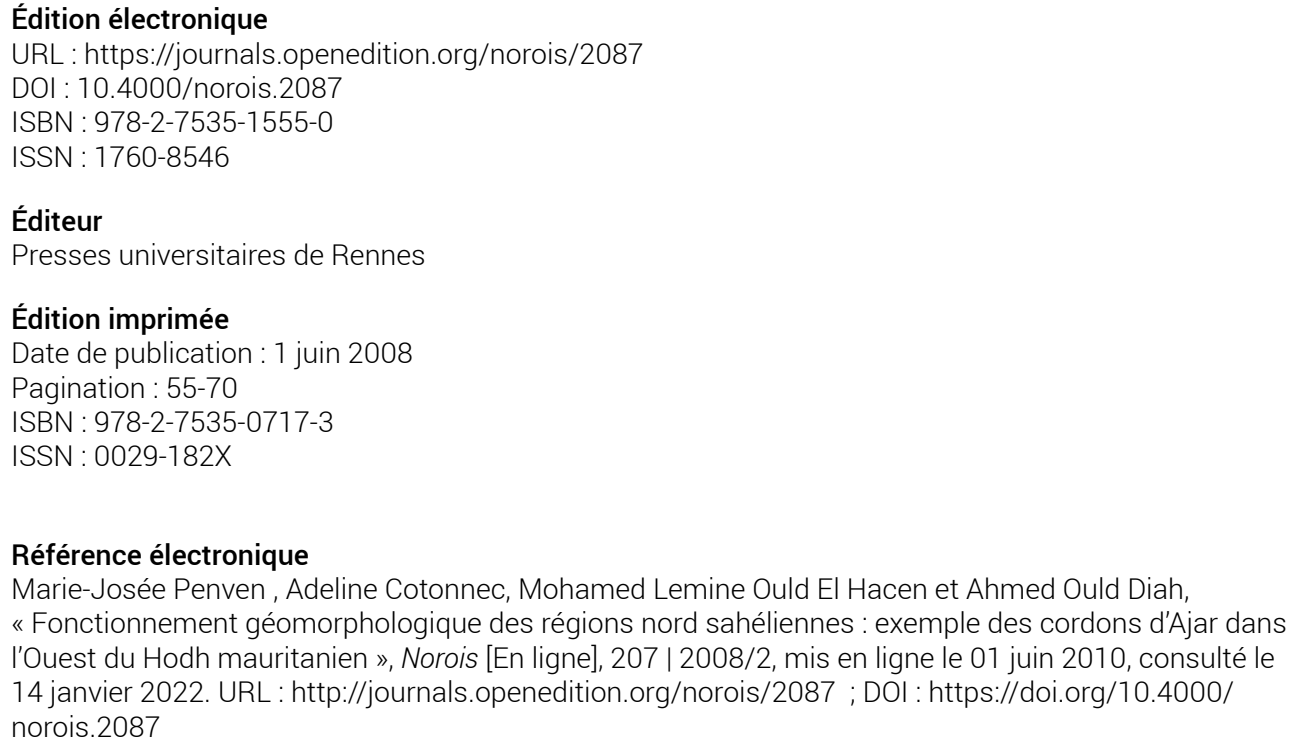




\title{
FONCTIONNEMENT GÉOMORPHOLOGIQUE DES RÉGIONS NORD SAHÉLIENNES EXEMPLE DES CORDONS D'AJAR DANS L'OUEST DU HODH MAURITANIEN
}

\author{
Marie-Josée Penven ${ }^{1}$, Adeline Cotonnec ${ }^{1}$, \\ Mohamed Lemine Ould El Hacen ${ }^{2}$, Ahmed ould diah ${ }^{1}$ \\ ${ }^{1}$ COSTEL - LETG UMR 6554 CNRS \\ (Université Rennes 2 Haute-Bretagne), \\ Place du Recteur-Henri-Le-Moal - 35043 REnNES cedex \\ 2 Département de GÉographie \\ (Université de Nouakchott), \\ BP 3329 - NouakchotT (Mauritanie) \\ marie-josee.penven@uhb.fr,adeline.cotonnec@uhb.fr
}

\begin{abstract}
RÉSUMÉ
Le Hodh mauritanien est une grande dépression de quelque $500 \mathrm{~km}$ d'ouest en est et de $250 \mathrm{~km}$ du nord au sud. Une couverture sableuse plus ou moins densément végétalisée s'étend au sud du $17^{e}$ parallèle. Elle laisse par place des sables vifs et affleurements rocheux. Dans un contexte climatique sec (200 à $300 \mathrm{~mm}$ de précipitations moyennes annuelles sur 3 mois), le système des cordons dunaires d'Ajar situé au sud de Kiffa (Hodh occidental) est analysé. Les indicateurs des processus hydriques et éoliens et leur enchaînement temporel à l'échelle annuelle sont mis en évidence ainsi que leurs interactions. Les différenciations spatiales des bilans géomorphologiques sont contrôlées par les organisations locales héritées dans un contexte régional situé à l'aval vent du système global éolien saharien.
\end{abstract}

Mots CLÉS : Sahel - Mauritanie - Processus hydriques - Processus éoliens - Interactions.

\section{ABSTRACT \\ Geomorphological processes in the north sahelian regions: the example of the Ajar aeolian sandy system in the West mauritanian Hodh}

The Hodh region is a $500 \times 250 \mathrm{~km}$ large depression in the south east of Mauritania. A sandy area is extending in the southern part and is covered by a variable density of grass vegetation. The studied region is located in the South of Kiffa in the western Hodh and it is characterized by a 9 months dry season and a short wet season and an average rainfall of 200 to $300 \mathrm{~mm}$. The analyse of the sandy system of Ajar shows the interaction of hydrological and aeolian process for scale time of the year. Geomorphological and sediment budgets varie a lot with sandy areas patchwork combinating relative stability or net exportation of sands. This patchwork is depending of geomorphic patterns in a down wind regional location.

KEY WORDS : Sahel - Mauritania - Hydrological Processes - Aeolian Processes - Interactions. 
Depuis la fin des années 1990, une amélioration des précipitations est enregistrée sur le Sahel occidental (Nicholson, 2005), permettant le développement de la couverture végétale herbacée et l'existence de ressources en eau superficielles, temporaires et souvent modestes (mares, petits barrages, cours d'eau) mais faciles d'accès pour le bétail. Cependant la couverture végétale dont le développement maximal se situe en août et septembre, ne constitue qu'une protection limitée contre les agents géomorphologiques, en raison d'une densité modérée et d'une existence provisoire. En effet, elle est d'une part soumise au pâturage, d'autre part atteinte par une sénescence rapide liée à l'arrivée de la saison sèche. Aussi les formations superficielles et les sols, dont la distribution résulte d'héritages anciens (pléistocènes et holocènes), sont-ils soumis alternativement ou en interaction, à l'échelle annuelle comme à des échelles décennales, à l'action des agents hydriques et éoliens dont l'efficacité dépend du contexte météorologique et reste à estimer.

S. M. Visser et al. (2004) notent que l'érosion éolienne (Tricart et Cailleux, 1969; Mainguet, 1995 ; etc.) et l'érosion hydrique (Collinet et Valentin, 1979; Chevallier et al., 1985; Mietton, 1988, Casenave et Valentin, 1989; Karambiri et al., 2003) sont généralement étudiées séparément alors que leur combinaison est essentielle dans le fonctionnement de ce type de milieu. Quelques auteurs traitent cependant de cet aspect soit en évoquant les vents violents précédant les précipitations (Mietton, 1988; Orange et al., 1998), soit en mettant en évidence le rôle des croûtes structurales dans la limitation de l'ablation éolienne (Hupy, 2004) ou dans la combinaison des processus éoliens et hydriques (Valentin, 1994; Visser et al. 2004). Ces derniers auteurs (Visser et al., 2004) soulignent l'intérêt de prendre en compte l'impact des rafales de vent dans les bilans d'érosion à l'échelle de la saison humide. Toutefois, Orange et al. (1998) montrent qu'à l'échelle des domaines soudaniens et sud sahéliens de l'Afrique de l'Ouest, seuls $25 \%$ des dépôts éoliens se font pendant la période humide. De fait, le bilan morphogénique (rapport ablation/sédimentation) dans ce type de milieu est la résultante, à l'échelle annuelle, de l'action locale (versants/bassins versants) des écoulements de surface et de l'action régionale et macrorégionale des vents.

L'objectif est ici de mettre en évidence, dans une région nord sahélienne, les fonctionnements géomorphologiques, en insistant sur l'enchaînement des processus hydriques et éoliens, dans une unité spatiale caractéristique. Les bilans géomorphogéniques peuvent être ainsi approchés à différentes échelles d'observation. Le champ d'application retenu est le système dunaire d'Ajar, situé à une trentaine de kilomètres au sud de Kiffa, dans le Hodh occidental mauritanien (fig. 1 et 2).

La méthode appliquée est celle de l'observation terrain à différentes échelles (du quadrat à l'unité géomorphologique élémentaire [sommet de cordon, dépression inter cordon, etc.]) le long de séquences définies à partir de l'analyse visuelle de compositions colorées effectuées sur des images Landsat ${ }^{1}$ datant de septembre 2001 et novembre 2002. La spatialisation des observations et leur positionnement dans le contexte général permettent une réflexion sur les bilans géomorphogéniques.

\section{Le cadre régional de l'étude}

La région étudiée se situe à l'Ouest du Hodh mauritanien, vaste dépression de $500 \mathrm{~km}$ d'ouest en est (entre 7 et $12^{\circ}$ de longitude est) et de quelques $250 \mathrm{~km}$ du nord au sud jusque la frontière malienne (entre 18 et $15^{\circ} 30^{\prime}$ de latitude nord). Elle forme une boutonnière délimitée par des talus d'un commandement de 200 à 250 m et occupée en son centre ouest par les plateaux démantelés de l'Affolé. Avec des altitudes moyennes comprises entre 100 et $200 \mathrm{~m}$, cette dépression appartient à la zone saharo-sahélienne (Biswas in Mainguet, 1995) s'étendant depuis les territoires arides sahariens au nord (100 à 150 mm et moins de 15 jours de pluie par an) jusqu'aux territoires subarides sahéliens au sud $\left(200\right.$ à $400 \mathrm{~mm}$ au sud du $17^{\mathrm{e}}$ parallèle et environ 20 à 25 jours de pluies annuelles). En opposition au nord de la dépression occupée par des sables vifs façonnés en dunes transverses, le sud du $17^{\mathrm{e}}$ parallèle se caractérise par une couverture sableuse végétalisée plus

1. Les images utilisées sont issues du site de l’Université du Maryland, Institute for Advanced Computer Studies (USA). 
Figure 1 : Les grandes unités physiographiques du Sud-Est mauritanien et la localisation de la zone d'étude

\section{Main physi-} ographic units in southeastern Mauritania and location of the studied zone

1 : plateaux dominant la dépression du Hodh. 2 et 3 : la dépression du Hodh (2: secteur à dominante rocheuse; 3 : les aires sableuses). 4 : les plateaux centraux de l'Affolé.

1: plateau overlooking the Hodh depression; 2: Hodh depression, rockdominated areas; 3 : Hodh depression, sanddominated areas; 4 : central plateau of Affolé.
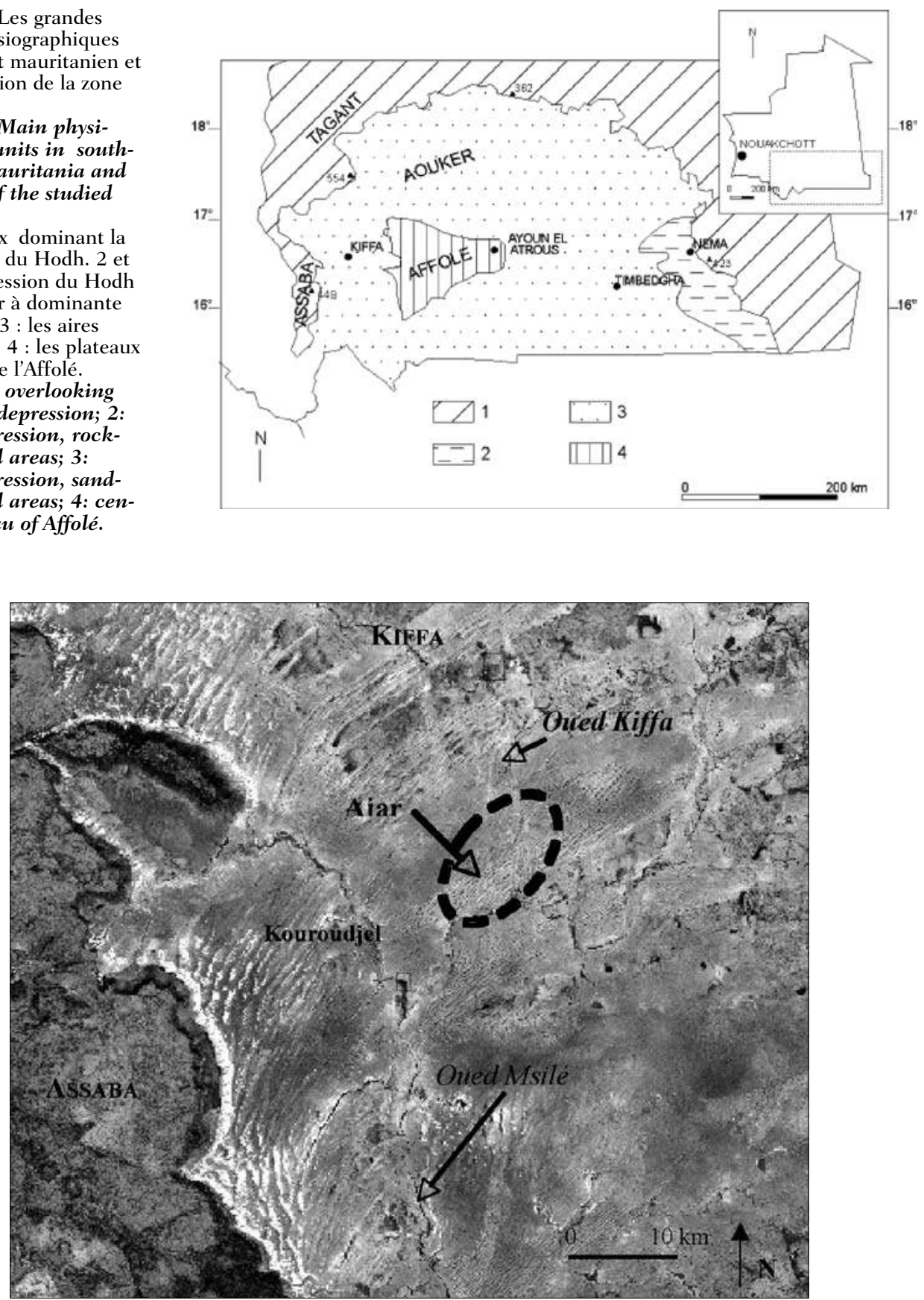

Figure 2 : Localisation du système dunaire d'Ajar dans la région de Kiffa (combinaison des canaux 2, 5 et 4 de Landsat TM, novembre 2002)

Location of the Ajar dune system, in Kiffa region (Landsat TM image of November 2002, composition with bands 2, 5 and 4) 
ou moins continue. Un réseau hydrographique à régime temporaire s'y développe, endoréique à l'est et au centre, exoréique à l'ouest. Cette région appartient au domaine de transition entre le domaine aride saharien et le domaine semi-aride sahélien.

Dans le secteur occidental, la région de Kiffa (fig. 2) ou plaine de «Regueïba » (Doutressoulle, 1947) constitue un couloir de direction méridienne, d'environ $50 \mathrm{~km}$ de large, entre le talus de l'Assaba à l'ouest et les plateaux de l'Affolé à l'est. Passant d'altitudes de 180-200 m au nord de Kiffa à environ $100 \mathrm{~m}$ au sud de Kouroudjel, cette unité possède un réseau hydrographique de direction générale nord-sud (l'Oued Kiffa-M'silé et ses affluents), incisé de 20 à 40 m selon les sections du cours d'eau. Sous affluent du fleuve Sénégal, cet axe hydrographique ne draine cependant pas l'ensemble de cette région : en effet la couverture sableuse façonnée ou non en une série de cordons dunaires introduit une multitude de petites unités hydrographiques endoréiques.

Les conditions climatiques de ce secteur du Hodh peuvent être précisées grâce aux données de la station météorologique de Kiffa. Comme tout le Sahel, cette région se caractérise par la faiblesse des précipitations annuelles, leur variabilité interannuelle. Le total moyen annuel, calculé sur la période 1940-2004, est de 293,4 mm (tableau 1a), avec une courte saison pluvieuse de 3 mois et 24 jours de pluies. Elles tombent sous forme d'averses de très forte intensité, parfois très abondantes (les $140 \mathrm{~mm}$ enregistrés à Timbedgha le 7 septembre 2003 dans l'est du Hodh illustrent ce type d'événement).

a) les précipitations

\begin{tabular}{|c|c|c|c|c|c|c|c|}
\hline \multirow{2}{*}{$\begin{array}{c}\text { Moyenne } \\
\text { annuelle } \\
(\mathrm{mm})\end{array}$} & \multicolumn{5}{|c|}{ Moyennes mensuelles (mm) } & \multirow{2}{*}{$\begin{array}{l}\text { Q 50 } \\
(\mathrm{mm})\end{array}$} & \multirow{2}{*}{$\underset{(\mathrm{mm})}{\mathrm{Q} 1 \text { et } \mathrm{Q}} 99$} \\
\hline & juin & juillet & août & sept. & oct. & & \\
\hline 293,40 & 20,90 & 65,00 & 111,80 & 73,00 & 17,30 & 274,00 & $\begin{array}{l}118,00 \\
534,00\end{array}$ \\
\hline
\end{tabular}

Série chronologique : 1940-2004, sources ONM

b) les températures moyennes $\left({ }^{\circ} \mathrm{C}\right)$

\begin{tabular}{|c|c|c|c|c|c|c|c|c|c|c|c|c|}
\hline janvier & février & mars & avril & mai & juin & juillet & août & sept. & oct. & nov. & déc. & moy. \\
\hline 23 & 25,9 & 29,1 & 33 & 35,8 & 36,2 & 33,3 & 31,7 & 32,0 & 32,2 & 28,15 & 23,8 & 30,1 \\
\hline
\end{tabular}

Série chronologique : 1970-2004, sources ONM

Tableau 1 : Les caractéristiques climatiques de la station de Kiffa Climatic characteristics in weather station of Kiffa

En année moyenne, juin reçoit les premières pluies avec des totaux modérés sur 1 à 4 jours. Juillet, août et septembre sont les plus arrosés avec un maximum d'août; octobre peut connaître des pluies d'arrière-saison. Les valeurs moyennes masquent une variabilité interannuelle très élevée avec un rapport d'environ 1 à 4 entre les valeurs extrêmes. La valeur de Q50, inférieure à la moyenne, traduit une dissymétrie dans la distribution interannuelle des précipitations au détriment des années humides.

La température moyenne annuelle, calculée sur 35 ans, est de $30{ }^{\circ} \mathrm{C}$ (tableau lb). L'amplitude annuelle modeste de $10^{\circ} \mathrm{C}$ dissimule des maxima moyens mensuels parfois élevés (juin 1995 : $44^{\circ} \mathrm{C}$ ). Le bilan hydrique calculé sur les mois humides (Atlas de Mauritanie, 1999) indique des conditions drastiques de vie : le déficit de 400 à $500 \mathrm{~mm}$ dans la région de Kiffa passe à 300$400 \mathrm{~mm}$ à proximité de la frontière malienne.

Le régime des vents (Ould Elhacen, 2000) est caractérisé, en saison sèche, par l'alizé continental avec au début de cette saison, son installation progressive tandis que se maintiennent des calmes fréquents, surtout en octobre. La période de décembre à mai voit la fréquence des vents de vitesse comprise entre 5 et $9,9 \mathrm{~m} / \mathrm{s}$ augmenter dès janvier (à Kiffa, $45 \%$ de l'ensemble 
des vents enregistrés à 12 heures) et ceux $>10 \mathrm{~m} / \mathrm{s}$ dès le mois suivant. Avec des vents encore violents ( $32 \%$ ont une vitesse $>5 \mathrm{~m} / \mathrm{s}$ ) mais multidirectionnels, le mois de mai marque une transition. L'hivernage voit s'imposer les vents de secteur sud et ouest avec une diminution nette des vents de vitesse $>5 \mathrm{~m} / \mathrm{s}$ et une augmentation des calmes. Septembre se caractérise par des vents multidirectionnels et modérés. L'efficacité morphogénique de l'alizé continental se situe entre janvier et juin, en terme de fréquence (janvier) ou en terme de vitesse (mai, juin). Selon l'Atlas de Mauritanie (1999), cette région enregistre en moyenne par an 50 à 100 jours de chasse sable et 100 à 150 jours de brumes sèches.

Le développement de la couverture végétale, conditionné par les précipitations irrégulières d'une année à l'autre et d'une sous région à une autre, est également contrôlé par la nature du substrat, rocheux ou sableux. Sur les accumulations sableuses (nappes sableuses, cordons), la couverture végétale est composée d'une steppe arborée à Acacias (raddiana, flava, senegalensis) et Balanites aegyptica, auxquels peut s'associer Leptadania pyrotechnica. Les herbacées (Cenchrus biflorus, Heliotropum bacciferum, Alysicarpus ovalifolius, etc.) ont un taux de recouvrement qui varie de 30 à 60-70\%, en fonction de l'histoire locale récente à l'échelle de la saison et de l'année (pluviométrie, pâturage). Dans les dépressions des couvertures sableuses (inter cordon, dépression fermée), la densité d'arbres est plus élevée et, dans certaines, est présent Acacia nilotica, indicateur de réserves hydriques plus importantes et d'ennoiements temporaires fréquents. Les sommets de certains cordons, mais aussi les abords des villages et points d'eau, sont dénudés, seuls des arbres y subsistent.

\section{L'organisation des cordons d'Ajar}

Les alignements de cordons (fig. 3 - planche VII). Situé au sud de Kiffa, l'ensemble des cordons, d'une longueur maximale de $12 \mathrm{~km}$ et d'une largeur de 2 à $4 \mathrm{~km}$, est délimité à l'est et au sud par la vallée de l'Oued Kiffa sur lequel se situe le lac Ajar. De direction nord-est - sud-ouest, les cordons forment un ensemble serré d'ondulations d'amplitude altitudinale de 6 à $16 \mathrm{~m}$. Les crêtes sont distantes de 150 à $450 \mathrm{~m}$, ménageant des cordons souvent dissymétriques aux flancs plus ou moins raides et des dépressions inter cordon plus ou moins évasées.

Le système en cordons, hérité d'une morphogenèse éolienne à bilan négatif, repose sur une paléo-topographie constituée d'un substratum de pélites siliceuses au modelé différencié et supportant localement une carapace ferrugineuse. Deux séries sableuses d'une épaisseur totale modeste $(6$ à $16 \mathrm{~m})$ composent les cordons : à la base des sables rubéfiés, compacts, à caractère limono-sableux ( $10 \%$ d'argile et 10 à $30 \%$ de limons), au-dessus des sables plus récents, légèrement limoneux. Une troisième série constituée de sables éoliens actifs couronne la partie sommitale de certains cordons.

Les inter cordons sont occupés par de longues dépressions, plus ou moins comblées de sédiments limono-sableux grisâtres et végétalisées. Elles peuvent être fermées et organisées en chapelets, séparées par des seuils soit sableux, soit rocheux et fréquemment recouverts d'un cailloutis de pélites et/ou de débris de carapace ferrugineuse. Elles peuvent également être reliées au réseau hydrographique régional grâce à des axes élémentaires. Ainsi se juxtaposent sur une superficie limitée deux systèmes, l'un endoréique, l’autre exoréique.

À l'échelle locale, des organisations diversifiées des unités sableuses. Trois types d'agencement se distinguent (fig. 4):

- Des cordons à sommets sableux mobiles, dissymétriques : du sommet à la base se succèdent 3 unités (fig. 4a):

1) les sables éoliens mobiles, de couleur ocre (5YR 6/8), bien triés (fig. 5), s'étendant sur une largeur de 3-4 m à une dizaine de mètres. La végétation se résume à quelques arbres et buissons isolés. 


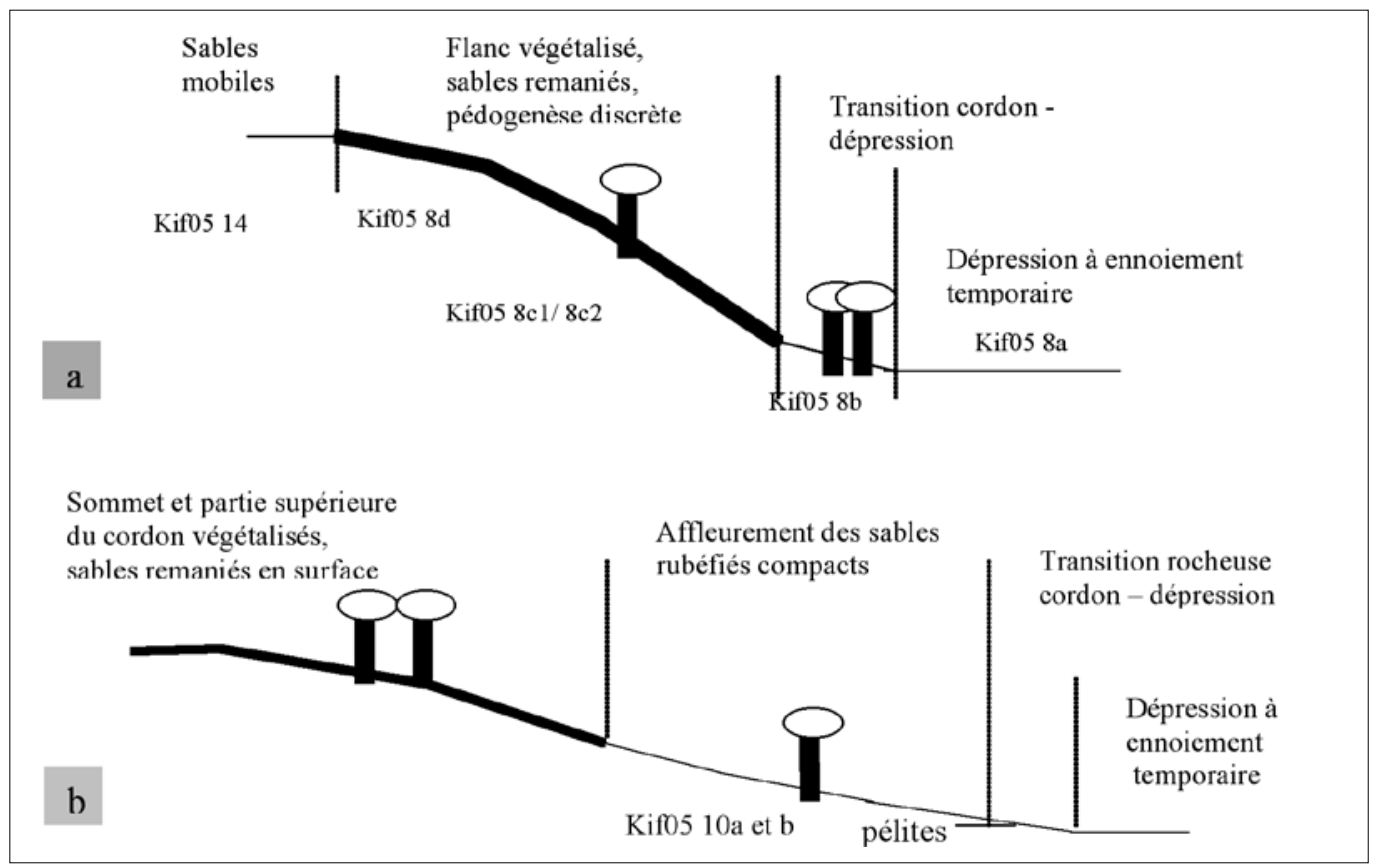

Figure 4 : Organisation des unités sableuses à l'échelle des cordons At the ridge scale: distribution of sandy units $\mathrm{a}$ : cordons à sommets sableux mobiles ; b : cordons à sables rubéfiés végétalisés. a: ridges with a mobile sandy crest; b: ridges with vegetation on rubefied sand.

2) le flanc du cordon dont la pente de quelques dizaines de mètres de long, atteint des valeurs de 5 à $15^{\circ}$ et où les sables sont recouverts d'une steppe claire parsemée de quelques arbres (Balanites aegyptica dominant associé à des acacias) très inégalement espacés (de quelques mètres à plusieurs dizaines de mètres). Le taux de couverture au sol par les herbacées est très inégal : avec 75 à 150 individus au $\mathrm{m}^{2}$, il varie de 20 à $70 \%$. En surface, 15 à $20 \mathrm{~cm}$ de sables remaniés et possédant des traits pédogéniques discrets reposent sur le matériel in situ (8 à $10 \%$ de limons, fig. 5).

3) la zone de transition entre cordon et dépression à ennoiement temporaire, à pente faible (2 à $3^{\circ}$ ) de 5 à $10 \mathrm{~m}$ de large, est constituées de sables grisâtres remaniés (10YR 6/3) légèrement limoneux (15 à $16 \%$ de limons, fig. 5), pulvérulents. Un alignement de Balanites aegyptica occupe cette unité de transition.

- Des cordons entièrement végétalisés. Généralement peu élevés, leur dissymétrie est atténuée et les valeurs de pente ne dépassent pas $10^{\circ}$. Le matériel constitutif sur leur partie supérieure et la végétation associée sont identiques à ceux du flanc de cordon précédemment évoqué. La partie inférieure est formée de sables rubéfiés sous une vingtaine de centimètres de sables remaniés et recouverts d'une steppe plus ou moins dense.

- Des cordons à affleurements de sables rubéfiés (fig. 4b). Leur forme générale est identique à celle des cordons précédents. Le sommet est constitué des mêmes sables légèrement pédogénisés en surface et recouverts par une steppe plus ou moins dense piquetée de Balanites aegyptica et d'acacias. La moitié ou le tiers inférieur du cordon laisse affleurer des sables rubéfiés (7,5 YR 5/4), compacts, fortement limoneux (fig. 6). Seuls quelques arbres subsistent ou encore des plaques d'herbacées sur des sables résiduels. Le contact avec la dépression se fait soit par l'intermédiaire d'un affleurement rocheux plus ou moins masqué par des sables, soit par une concavité façonnée dans les sables qui se poursuivent dans l'inter cordon. 
Figure 5 : Granulométrie des sables d'un cordon au Nord du système d'Ajar (localisation des échantillons sur la figure 4a)

Grain size diagram for the sand of a ridge, North of the Ajar system (These samples are located on fig. 4a)

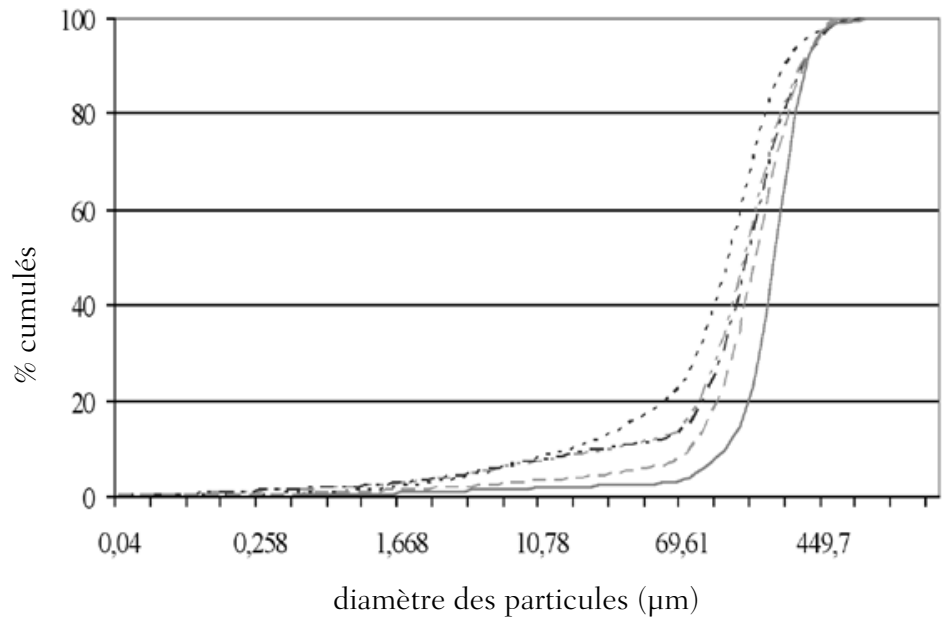

Dépression intercordons (Kif05 8a )

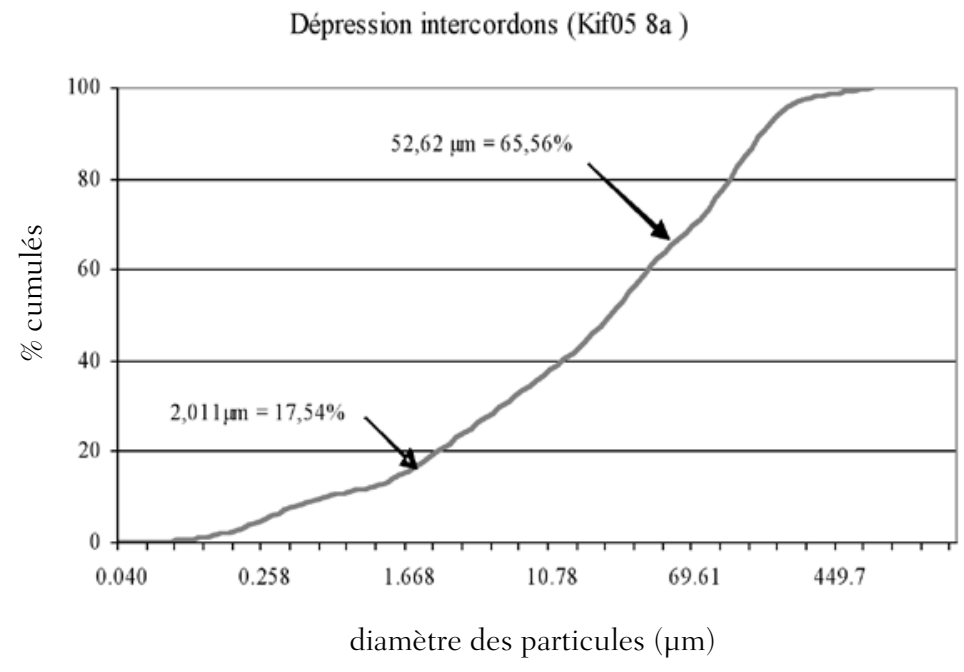

diamètre des particules $(\mu \mathrm{m})$
Figure 6 : Granulométrie du remplissage sédimentaire d'une dépression inter cordon (localisation des échantillons sur la figure 4a)

Grain size diagram for the sediment infilling an inter ridge depression (this sample is located on fig. 4a)

Les dépressions fermées sont colmatées en tout ou partie par des limons sableux grisâtres (fig. 7) où, selon la fréquentation du bétail, dominent soit des polygones de dessiccation, soit des traces de piétinement.

\section{Les indicateurs des processus géomorphogéniques}

De nombreux indicateurs de l'action des agents géomorphogéniques s'observent aussi bien en saison sèche qu'en saison humide. Quels qu'ils soient (vent, écoulement des eaux), leur efficacité dépend en partie des caractéristiques des stocks sableux disponibles et de l'état de la couverture végétale. Ce sont donc les états de surface liés aux agents eux-mêmes mais aussi à l'utilisation de l'espace par les populations qui en sont les indicateurs. Deux types d'état de surface sont à 


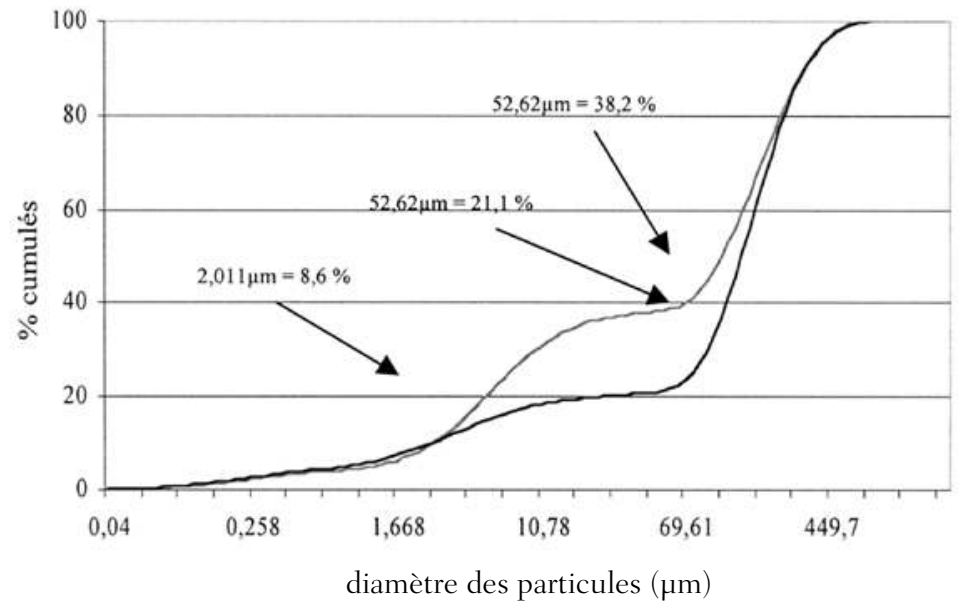

Figure 7 : Granulométrie des sables rubéfiés (localisation des échantillons sur la figure 4b)

Grain size diagram for the rubefied sands (these samples are located on fig. 4a)

l'origine de la mobilisation des particules sableuses par les agents géomorphogéniques, induisant des processus diversifiés.

\section{LES ÉTATS DE SURFACE OBSERVÉS}

- Des crô̂tes de battance. Lors de la saison des pluies, sur les sables vifs des sommets de cordon, ne se développe qu'un film réduisant la rugosité des sables mais ne permettant guère le ruissellement des eaux. En revanche, sur les parties de cordon végétalisé et sur les sables rubéfiés, les teneurs en limons permettent la formation de croûtes de battance (dont certaines à Cyanophycées) qui diminue les capacités d'infiltration des eaux et donc favorise les possibilités de ruissellement (photo 1a - planche VII). Très fragiles, elles sont facilement détruites par le piétinement du bétail (photo $1 \mathrm{~b}$ - planche VII).

- Des structures particulaires. Dans la région de Kiffa, une pression pastorale forte s'exerce en certaines périodes de l'année (saison humide et premiers mois de la saison sèche). Outre la réduction de la couverture végétale par la sécheresse et par le pâturage (photo 2a), la présence du bétail provoque un piétinement des sables. Ce piétinement occasionne une dispersion des sables et limons, induisant l'existence d'une structure particulaire (photos $2 \mathrm{~b}$ et c) et donc la présence d'un stock disponible pour les agents géomorphologiques. Seules les formations rubéfiées compactes ne subissent pas ce processus de dispersion (photos 3a et b-planche VIII).

Enfin, il est à noter que le passage répété du bétail en troupeaux (notamment les déplacements réguliers de l'aire de pâture à un point d'eau) laisse des traces soit en bandes isolées larges de 20-30 cm se répétant à flanc de cordons, soit en bandes larges de quelques mètres et recoupant souvent perpendiculairement les cordons (photo 4). Ces secteurs représentent des aires de fragilité sur le plan géomorphogénique.

\section{LES INDICATEURS DES PROCESSUS ACTUELS}

Les processus à l'origine du fonctionnement actuel relèvent à la fois des agents éolien et hydrique dont les marques sur les modelés sont plus ou moins spectaculaires. Plusieurs mécanismes peuvent être mis en évidence.

- Des indicateurs du façonnement par le vent sont concentrés sur les sommets de cordon occupés par les sables vifs. Des cuvettes de déflation s'observent autour des arbres déchaussés (photo 5 a) mais aussi des affleurements de sables lités affectés par la corrasion. Les accumulations se 


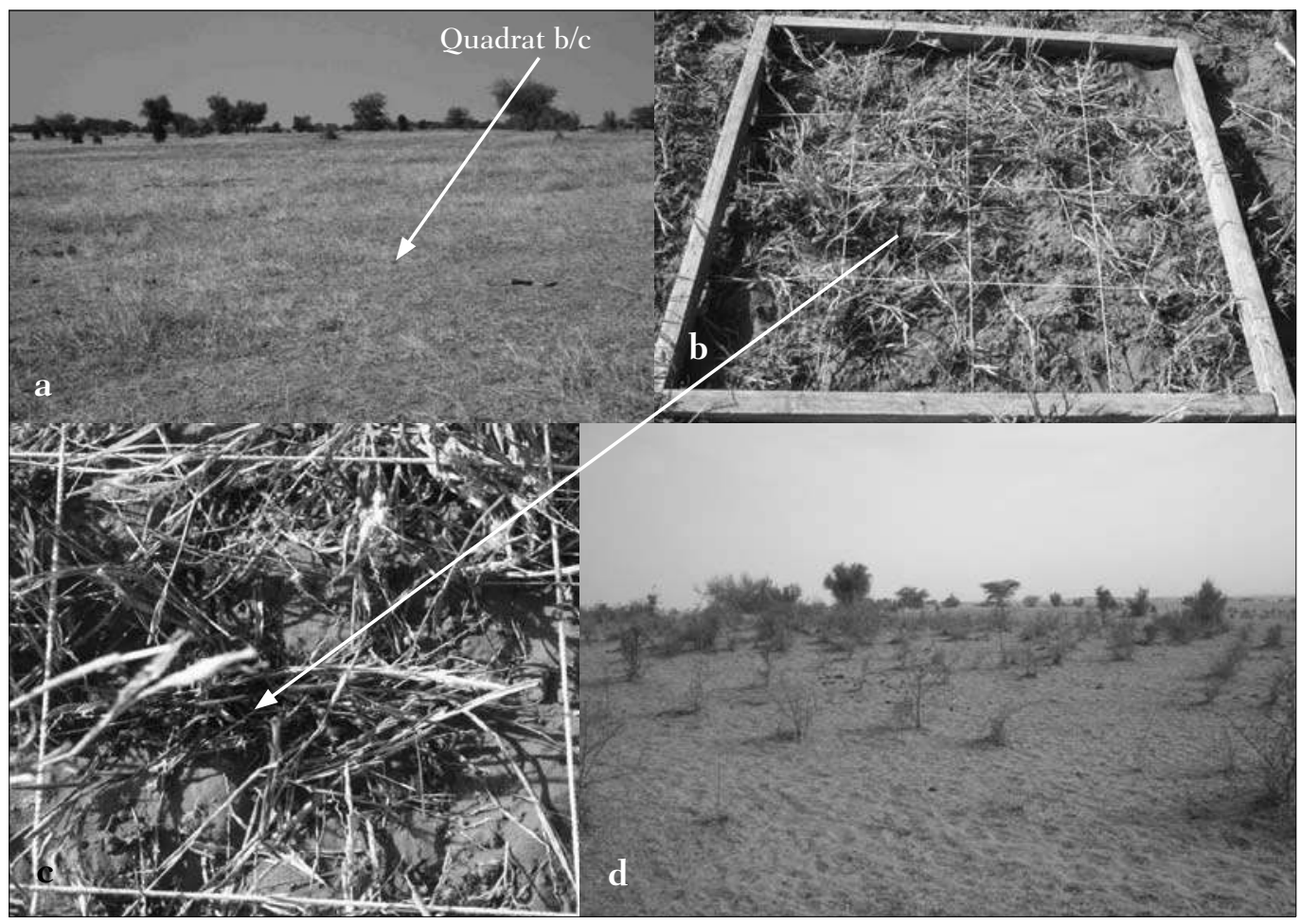

Photos 2 : Couverture au sol par la végétation sur un cordon et structure particulaire

a : le cordon, une couverture au sol inégale; b et c : couverture partielle par une végétation sénescente pâturée et structure particulaire des sables piétinés; $d$ : structure particulaire généralisée au cordon des sables piétinés après réduction de la couverture herbacée.

Vegetation soil cover on a sand ridge and particular structure

$a:$ uneven vegetation on the sand ridge; $b, c:$ uneven cover by grazed senescent vegetation and particular structure of stamped sands; $d$ : generalized particular structure on sand ridge after reduction of grass vegetation.

Photo 4 : Effets du passage répété du bétail passage way

Impact of cattle

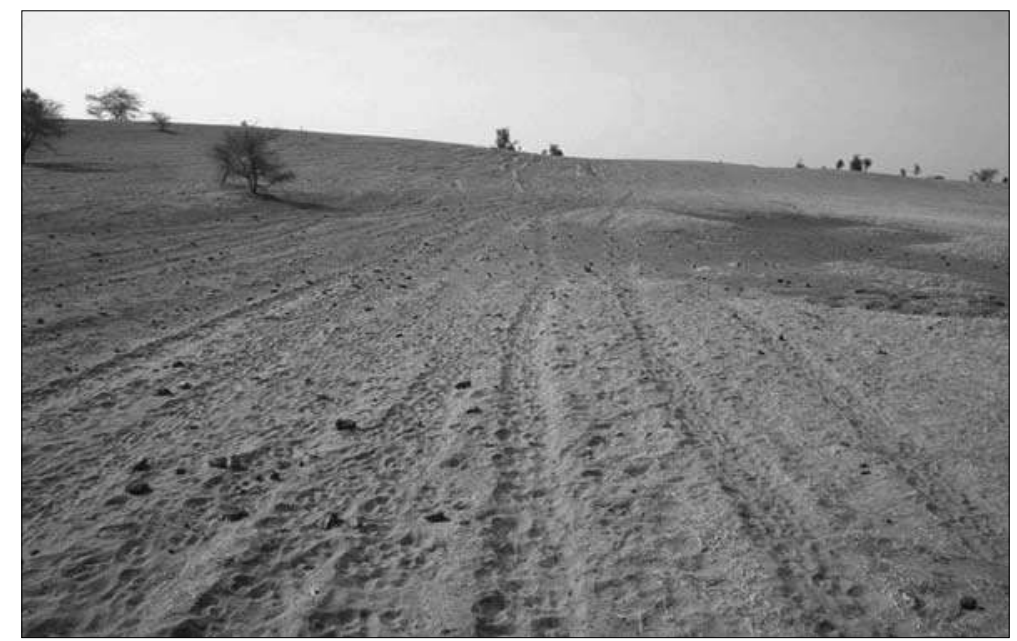




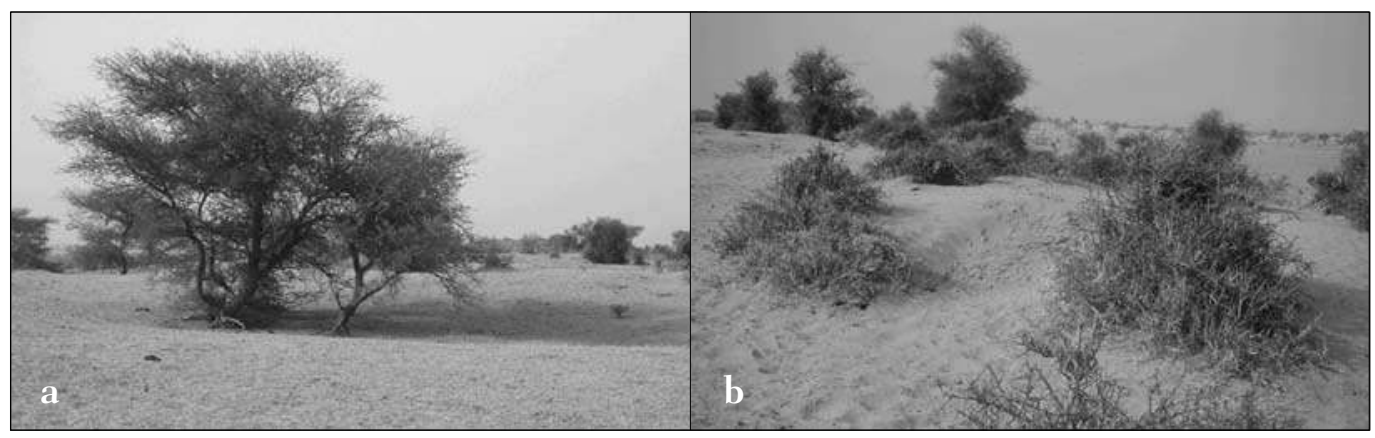

Photos 5 : Actions éoliennes sur des sommets de cordon

a : cuvette de déflation et déchaussement d'un acacia; b : accumulations sableuses en relation avec des buissons de Balanites aegyptica.

Aeolian processes on the top of a sand ridge

$a$ : deflation hole around an acacia; $b$ : sand deposits linked with a bush of Balanites aegyptica.

manifestent sous forme de bourrelets (photo $5 \mathrm{~b}$ ) à l'aval vent de buissons (les nebkas sont exceptionnelles) ou encore de crêtes vives dissymétriques en saison sèche.

Des voiles sableux millimétriques à centimétriques, témoignant d'une aspersion éolienne, jonchent les croûtes de battance subsistant entre les touffes herbacées.

- De nombreuses plages dénudées s'observent:

- les plages de cailloutis en position d'inter cordon constituant de petits regs de quelques centaines de $\mathrm{m}^{2}$ (photo 6a-planche VIII) : très dense, éolisé, le cailloutis est serti par des sables limoneux où la croûte de battance (photo $6 \mathrm{~b}$ - planche VIII) se maintient longtemps pendant la saison sèche à cause de l'assemblage protecteur que forme le cailloutis face à la déflation mais aussi parce que ces surfaces ne sont que peu fréquentées par le bétail.

- sur les cordons végétalisés, elles ont une dimension variable. Les petites ont une superficie moyenne d'1 à $2 \mathrm{~m}^{2}$, aux contours irréguliers; elles forment des cuvettes légèrement déprimées (une dizaine de centimètres) et sont toujours occupées par des sables pulvérulents piétinés. L'origine semble bien être le piétinement du bétail qui détruit localement le couvert herbacé. Formées soit pendant la saison des pluies, soit en début de saison sèche quand les troupeaux sont encore présents, ces plages représentent des aires de déflation potentielles pendant la saison sèche. La question de leur pérennité se pose à l'échelle interannuelle, leur revégétalisation par ensemencement par le vent étant possible.

Les plus grandes, d'ordre décamétrique (photo 7), mitent la steppe et ont un caractère permanent. La déflation éolienne est relayée par l'érosion hydrique en saison humide.

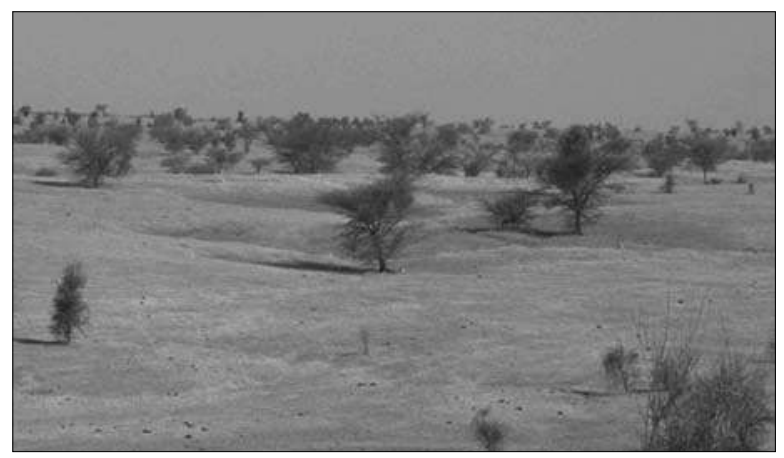

Photo 7 : Plages décamétriques dénudées Decametric bared areas 
- Des rigoles et ravines, associées ou non à des cônes d'épandage, sont observées sur les flancs des cordons.

Sur les cordons végétalisés, elles ne sont pas généralisées mais suffisamment nombreuses pour démontrer l'importance du processus de ruissellement concentré. La logique de leur distribution dans l'espace n'a pu être mise en évidence pour l'instant. L'organisation reste toujours la même : dans la partie amont, une croûte de battance généralisée en saison des pluies et quelques rigoles, dans la moitié ou le tiers inférieur une ravine de 20 à $40 \mathrm{~cm}$ de profondeur caractérisée par une érosion régressive (photos $8 \mathrm{a}$ et $\mathrm{b}$ ).

En revanche, sur les affleurements dénudés des sables rubéfiés, les rigoles et ravines (photo 8c), séparées par des surfaces battantes, sont généralisées et forment un réseau hiérarchisé arborescent. Si les rigoles ne font que quelques centimètres de profondeur, les ravines atteignent en moyenne 20 à $40 \mathrm{~cm}$, parfois 70 à $80 \mathrm{~cm}$. Elles sont fréquemment connectées à un réseau élémentaire sur les sables végétalisés situés en amont. La ravine principale est généralement connectée à un cône d'épandage où la densité des herbacées est faible (photo 8d). Les lits de ces ravines, dominés par des berges à vif, sont encombrés de sables issus des écoulements mais aussi des redistributions par le vent.

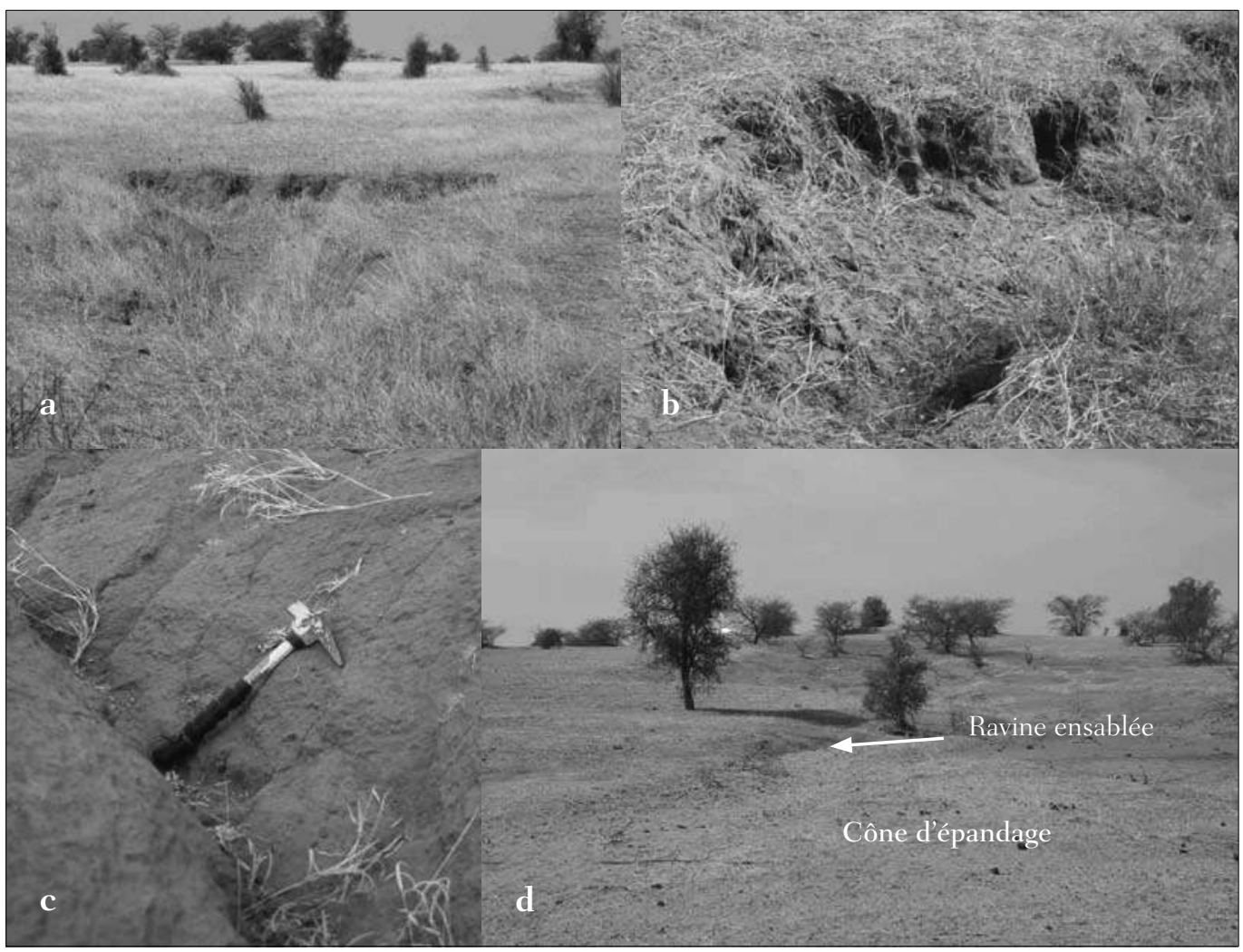

Photos 8 : Incisions dans les cordons

a et $\mathrm{b}$ : amont d'une ravine sur cordon végétalisé; $\mathrm{c}$ : rigole dans les sables rubéfiés; $\mathrm{d}$ : réseau hiérarchisé et cône d'épandage aval

Incisions on sand ridge

a, b: gully on a vegetated up ridge; $c$ : rill in the rubefied sands; $d$ : rill pattern and fans sands on the bottom of the ridge. 
- Les témoins des écoulements et concentrations des eaux dans les inter cordons

Les dépressions fermées sont alimentées :

- soit par le ruissellement diffus formé sur les cordons et dont témoignent les accumulations sableuses à leur pied,

- soit directement par des cônes d'épandages liés à des ravines,

- soit par des chenaux encaissés de quelque 10 à $40 \mathrm{~cm}$, larges de 50 à $120 \mathrm{~cm}$ dont le fond ensablé témoigne de la charge particulaire des eaux (photos 9a et b),

Sur la périphérie du système, certains inter cordons se caractérisent par une grande largeur et par la présence d'un substrat rocheux affecté par un système de pentes dont les valeurs sont comprises entre 1 et $4-5^{\circ}$. Des sables remaniés peuvent y former une fine pellicule. L'amont d'un réseau hydrographique élémentaire temporaire (photo 10) se raccordant à l'Oued Kiffa ou au Lac Ajar s’y incise.

De façon générale, les modelés issus des mécanismes éoliens et hydriques constituent une mosaïque fine témoignant d'une imbrication des agents érosifs dans le temps et l'espace.

\section{L'enchaînement des processus}

En début de saison sèche, la végétation herbacée offre une protection pendant un nombre de semaines varié dépendant de l'hivernage précédent et de la pression pastorale, dans un contexte

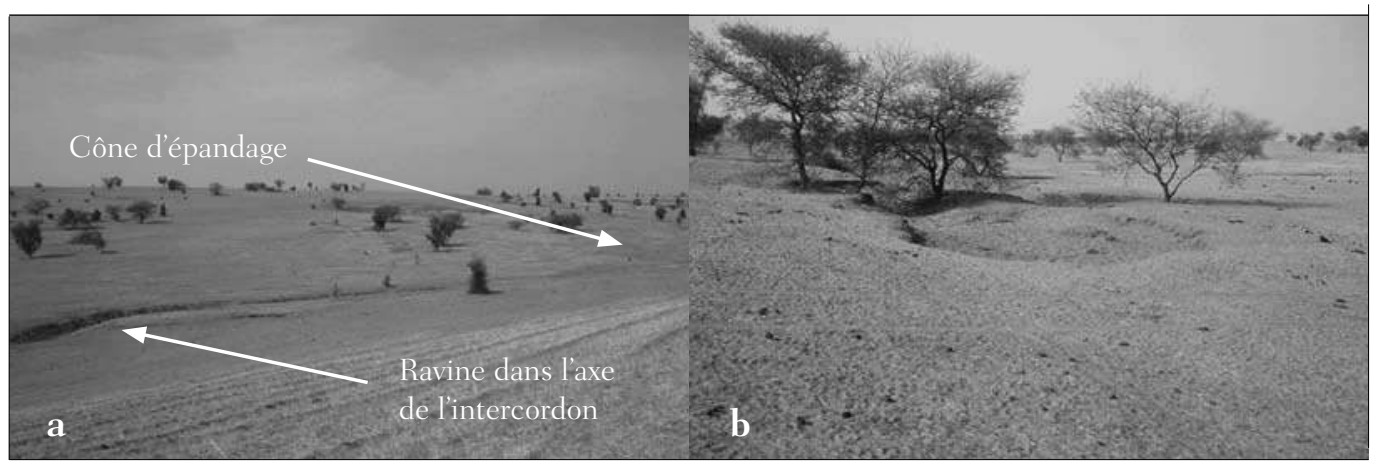

Photos 9 : Écoulements concentrés dans les inter cordons

Concentrated water flow on an inter ridge

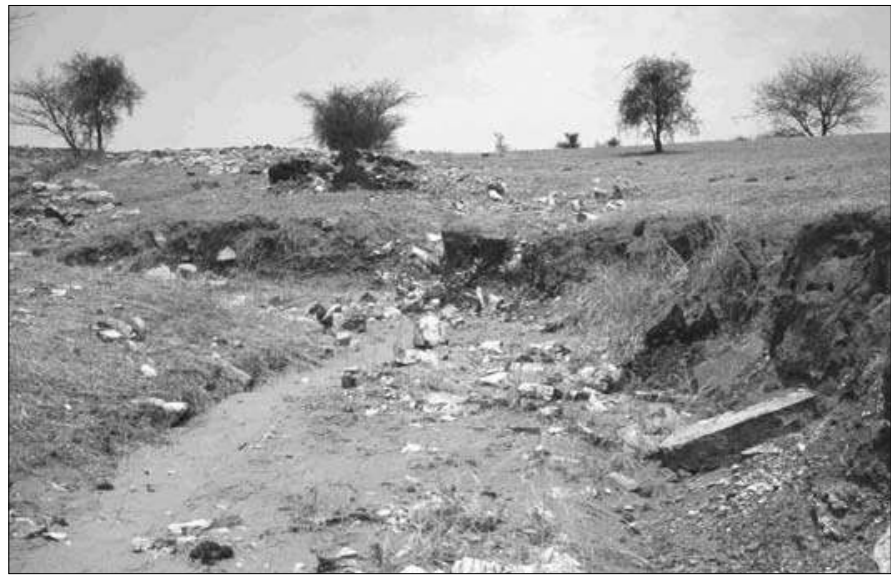

Photo 10 : Amont d'un axe hydrographique élémentaire

part of a catchment 
éolien généralement peu efficace. À partir de décembre-janvier, la mosaïque végétale résultant d’un inégal pâturage des troupeaux, associée à des états de surface particulaires, induit une réponse différenciée à l'action éolienne. À ce moment de l'année, on note de nombreux indices de son action dans les secteurs pâturés : cuvettes de déflation, voiles de sables d'une épaisseur d'ordre centimétrique, laissant supposer une aspersion éolienne. Sur les sommets des cordons, les remaniements éoliens se manifestent rapidement : ripple-marks, crêtes dissymétriques, déflation.

Au fur et à mesure que se déroule la saison et que le couvert végétal s'éclaircit, l'action éolienne se renforce. Les observations permettent d'envisager les effets différenciés de l'action éolienne en se fondant sur le modèle théorique de l'action éolienne (Mainguet, 1995). Les fonds inter cordons indemnes de toute accumulation traduisent une circulation des flux éoliens avec pour seul effet un entretien du modelé, la déflation étant modérée faute d’un stock de particules mobilisables (sables limoneux gris compacts des dépressions, plans caillouteux). Les seules accumulations observées affectent certains sommets de cordon, mais elles sont associées à des formes de déflation signalant des relais à grande échelle, la taille des sables indiquant un déplacement par roulement ou saltation. Les flancs de cordon indemnes d'accumulations sont parsemés de petites cuvettes de déflation mais les arbres ne sont pas déchaussés. Il semblerait que les flancs des cordons soient soumis à un travail en relais déflation/transfert/dépôt dont le bilan géomorphogénique est à peu près équilibré.

Les accumulations piégées dans les incisions liées à l'érosion concentrée, quelle que soit leur échelle, témoignent d'une alimentation par les vents et constituent des stocks disponibles lors de la saison humide suivante.

À la fin de la saison sèche, cet ensemble de cordons se caractérise par une couverture herbacée profondément dégradée, laissant place à un paysage minéral de sables associés à des plages caillouteuses et des cuvettes de sables limoneux gris compacts dans les inter cordons. Seul subsiste un piqueté d'arbres n'offrant aucune protection au sol.

La saison des pluies commence par des précipitations isolées peu abondantes permettant l'humectation des formations sableuses et le développement initial d'une croûte de battance. Alors que les précipitations continuent, la végétation herbacée se développe sur le flanc des cordons et dans les dépressions sableuses, la croûte de battance s'y parachève tandis que, sur les sommets indemnes de végétation protectrice, elle ne constitue qu'un film. Cette généralisation de la battance permet la réduction de l'infiltration et le développement d'écoulements superficiels. Par ailleurs, lorsque les surfaces caillouteuses présentes dans les inter cordons sont suffisamment vastes et à légère pente, des ruissellements à caractère élémentaire peuvent se former sur ces surfaces vite imperméabilisées (Albergel et al., 1992).

À l'échelle du cordon - inter cordon, sur les sommets des cordons, les eaux de pluie redistribuent les sables, adoucissant progressivement le modelé en dômes émoussés. Sur les flancs des cordons végétalisés, selon l'ampleur des averses, le ruissellement peut aller du ruissellement diffus élémentaire au ruissellement en nappe entre les touffes de végétation, ou encore former un micro-réseau d'incisions, exploitant les formes d'érosion héritées. Sur les sables rubéfiés dénudés et à croûte de battance permanente, les écoulements sont alimentés par ceux produits en amont sur la partie végétalisée et ceux engendrés sur les sables rubéfiés eux-mêmes. Ils se concentrent en un réseau hiérarchisé qui alimente un cône d'épandage, raccord avec la dépression inter cordon.

Ces ruissellements associés à des eaux en provenance des plans caillouteux, viennent alimenter en eau et particules les dépressions fermées, c'est-à-dire des aires de stockage, qui se transforment en mares ou lacs temporaires. Le système est fermé.

Dans les inter cordons connecté au réseau hydrographique élémentaire, les écoulements s'organisent depuis les cordons jusqu'au talweg. Le ruissellement se produit à la fois sur le système sableux de la même façon que ci-dessus et sur les plans caillouteux. Diffuses, en nappes ou concentrées, les eaux rejoignent la ravine dans l'axe de la dépression. L'érosion régressive y est active favorisant la mobilisation des particules sableuses et le recul des stocks sableux. Connecté au réseau hydrographique régional, le réseau élémentaire assure la continuité spatiale entre système local (aire 
de production) et système régional et il permet le transfert des sables vers les lits majeurs où les crues assurent une redistribution à l'échelle régionale après une ou plusieurs phases de stockage. Le système est ouvert, il assure l'exportation des eaux et particules hors du système.

\section{Conclusion}

De façon générale, les formes de l'activité éolienne sont concentrées dans l'espace (sommets de cordon sableux). Ailleurs, elles sont discrètes dans une région où le nombre de jours de vents de sables annuels est de 50 à 100. Les vents actifs de janvier à juin sont à l'origine d'une aspersion modeste (voiles sableux), de la prise en charge des sables dénudés à structure particulaire acquise sous l'action du piétinement, et de l'entretien des espaces inter cordons. Dans l'état des connaissances actuelles, il semblerait que les bilans soient à tendance équilibrée (entretien des inter cordons, redistribution en relais sur les sommets des cordons, grande discrétion des formes d'ablation éolienne caractérisées sur les flancs des cordons), cependant cet équilibre est fragile. Les vents assurent la redistribution locale (à grande échelle) ou régionale des particules, ce secteur appartenant, sur le plan éolien, à une aire de transfert dans le système régional. Toutefois, à l'échelle du Système Global d'Actions Éoliennes (Mainguet, 1995), cette région se situe à l'aval vent du système, donc en secteur d'accumulation. Néanmoins le caractère de couloir de la Regueïba de Kiffa (échelle moyenne) et multicouloirs de l'ensemble d'Ajar (grande échelle) assure un fonctionnement en venturis emboîtés, induisant le transfert des sables, si ce n'est leur ablation.

Les formes d'érosion hydrique, quant à elles, sont multiples et démontrent que des précipitations modestes (de l'ordre de $290 \mathrm{~mm}$ ) réparties sur 3 mois permettent une efficacité morphogénique dans une région où la couverture végétale steppique se caractérise par un taux de recouvrement modéré au sol. L'érosion hydrique provoque une redistribution locale des particules sableuses sur une partie du système des cordons d'Ajar lorsque les inter cordons sont fermés; en revanche la connexion avec le réseau hydrographique régional permet, lors des crues annuelles, l'évacuation des sables et leur exportation à l'échelle macrorégionale. Leur exportation vers l'aval du bassinversant dépend, à chaque saison humide, de la puissance des crues. Ainsi se juxtaposent à l'échelle du système d'Ajar deux fonctionnements, l'un endoréique, assurant le maintien des stocks sableux sur place, l'autre exoréique. La figure 8 montre que les aires d'exportation potentielles des sables via le réseau hydrographique représentent une superficie notable dans une région où à l'évidence les apports sableux par aspersion éolienne sont modestes.

Figure 8 : Les aires d'appauvrissement en particules sableuses par exportation via le système hydrographique élémentaire des cordons d'Ajar (a : aire endoréique ; $\mathrm{b}$ : aire exoréique)

Areas of sand loss

Sand is exported by the elementary hydrographic system of the Ajar ridges, (a: endoreic; b: exoreic)

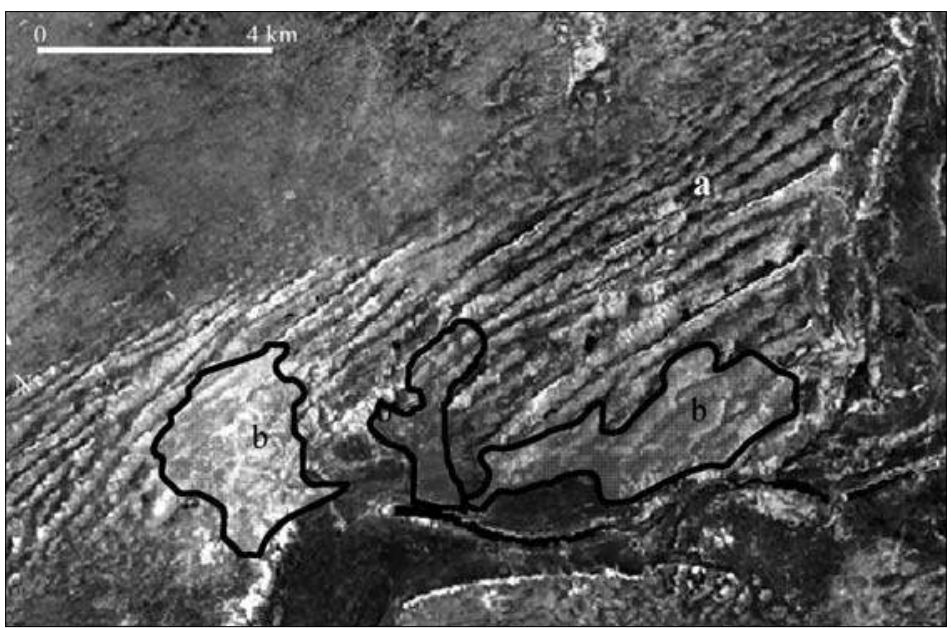


Il semble donc qu'une partie du système est soumise à des redistributions locales non négligeables pouvant entraîner des réorganisations des stocks sableux avec des aires de départ et d'accumulation, mais assurant le maintien des stocks sableux. En revanche une partie du système, soumis aux mêmes alternances de processus, se caractérise par des bilans négatifs, l'érosion hydrique assurant une perte en sables définitive et donc un appauvrissement en ressources végétales.

Le secteur d'Ajar se caractérise par une fine mosaïque d'unités et sous unités juxtaposées, marquées par des processus variés. Cependant ces juxtapositions ne doivent pas masquer les interactions existant entre les deux systèmes morphogéniques façonnant alternativement ce secteur au cours de l'année. Les interactions se situent sur 2 plans : 1) la production et la mise mutuelle à disposition de stocks sableux par les agents géomorphologiques se succédant dans le temps et l'espace; 2) l'exploitation mutuelle de modelés créés par l'un des deux agents, cette dernière interaction engendrant ou un ralentissement ou une accélération des processus. Par exemple, la présence des sables éoliens piégés dans des ravines et pris en charge par les eaux concentrées diminue la vitesse d'approfondissement des ravines, augmente la charge solide des eaux et favorise le fonctionnement en cônes d'épandage où l'ampleur des volumes de sables déposés limite le développement végétal, donc y favorise l'érosion éolienne!

Ces redistributions locales et exportations, associées aux apports par l'alizé continental, donnent de nombreuses possibilités de combinaisons et la mosaïque qui en résulte laisse deviner des bilans diversifiés et des réponses différentes en fonction des systèmes fonctionnels. Dans une région où la couverture sableuse est modeste, le fonctionnement géomorphologique conditionne la capacité de maintien des pâturages. Les vitesses d'évolution sont donc à préciser mais aussi les volumes de sables activés chaque année.

\section{Bibliographie}

Albergel (A.), Casenave (A.), Ribstein (P.) et Valentin (C.), 1992. - « Aridité climatique, aridité édaphique; étude des conditions de l'infiltrabilité en Afrique tropicale sèche », dans LE FLOC'H (E.) et al., L'aridité, une contrainte au développement, Paris, Éditions de l'ORSTOM, coll. « Didactiques », p. 123-130.

Atlas de Mauritanie, 1999. - Migrations et gestion des territoires, Université de Nouakchott/Université de Rouen, Coopération française, 12 planches, 1 fascicule, $64 \mathrm{p}$.

Casenave (A.), Valentin (C.), 1989. - Les états de surface de la zone sahélienne. Influence sur l'infiltration, Paris, ORSTOM, coll. « Didactiques », 229 p.

Chevallier (P.), Claude (J.), Pouyaud (B.), Bernard (A.), 1985. - Pluies et crues au Sahel. Hydrologie de la Mare d'Oursi (Burkina Faso). 1976-1981, Paris, Éditions de l'ORSTOM, 267 p.

Collinet (J.), VAlentin (C.), 1979. - «Analyse des facteurs intervenant sur l'hydrodynamique superficielle. Nouvelles perspectives : applications agronomiques », Cahiers de l'ORSTOM, série Pédologie, 17 (4), p. 285-328.

Doutressoulle (G.), 1947. - L'élevage en Afrique occidentale française, Paris, Larose, 298 p.

HuPY (J. P.), 2004. - «Influence of vegetation cover and crust type on wind-blown sediment in a semi-arid climate », Journal of Arid Environments, 58, p. 167-179.

Karambiri (H.), Ribolzi (O.), Delhoume (J.-P.), Ducloux (J.), Coudrain-Ribstein (A.), Casenave (A.), 2003. - « Importance of soil surface characteristics on water erosion in a small grazed Sahelian catchment », Hydrological processes, 17, 8, p. 1495-1507.

Mainguet (M.), 1995. - L'homme et la sécheresse, Paris, Masson, 335 p.

Mietton (M.), 1988. - Dynamique de l'interface lithosphère-atmosphère au Burkina Faso. L'érosion en zone de savane, Caen, Editec, vol. 1, 511 p.

NaHAL (I.), 2004. - La désertification dans le monde. Causes, processus, conséquences, lutte, Paris, L'Harmattan, coll. « Biologie, écologie, agronomie», 150 p. 
Nicholson (S.), 2005. - « On the question of the "recovery" of the rains in the West African Sahel », Journal of Arid Environments, 63, p. 615-641.

Orange (D.), Feizoure (C.), Guimba (C.), 1998. - « Erosion éolienne et apports géochimiques au sol par les poussières atmosphériques en Afrique de l'Ouest et Centrale », ORSTOM Actualités, n 56, spécial Érosion, p. 11-12.

Ould El Hacen (M.-L), 2000. - La route de l'Espoir, Thèse, Université de Reims Champagne Ardenne. $229 \mathrm{p}$

Ould Diah (A.), 2006. - Les grandes unités paysagères du Lac d'Ajar (Hodh Occidental, Mauritanie) : une approche spatiale et temporelle, Mémoire de Master 2, Rennes 2, $17 \mathrm{p}$.

SirCoulon (J.), 1992. - "Caractéristiques des ressources en eau de surface en zones arides de l'Afrique de l'Ouest », dans Le Floc'H (E.) et al., L'aridité, une contrainte au développement, Paris, Éditions de l'ORSTOM, coll. «Didactiques », p. 53-68.

Tricart (J.), Cailleux (A.), 1969. - Traité de géomorphologie. Le modelé des régions sèches, Paris, Sedes, t. IV, $472 \mathrm{p}$.

Valentin (Chr.), 1994. - « Sécheresse et érosion au Sahel », Sécheresse, 5 (3), p. 191-198.

Visser (S. M.), Sterk (G.), Ribolzi (O.), 2004. - « Techniques for simultaneous quantification of wind and water erosion in semi-arid regions », Journal of Arid Environments, 59, p. 699-717.

\section{Remerciements}

Cette étude a été menée dans le cadre d'une recherche en réseau «Environnement et Développement durable » de l'AUF, convention Prog.D. 2092RR623.

Cet article a été reçu le 30 juin 2007 et définitivement accepté le 3 mars 2008. 
Planche VII (Marie-Josée PENVEN et al. - Fonctionnement géomorphologique des régions...)

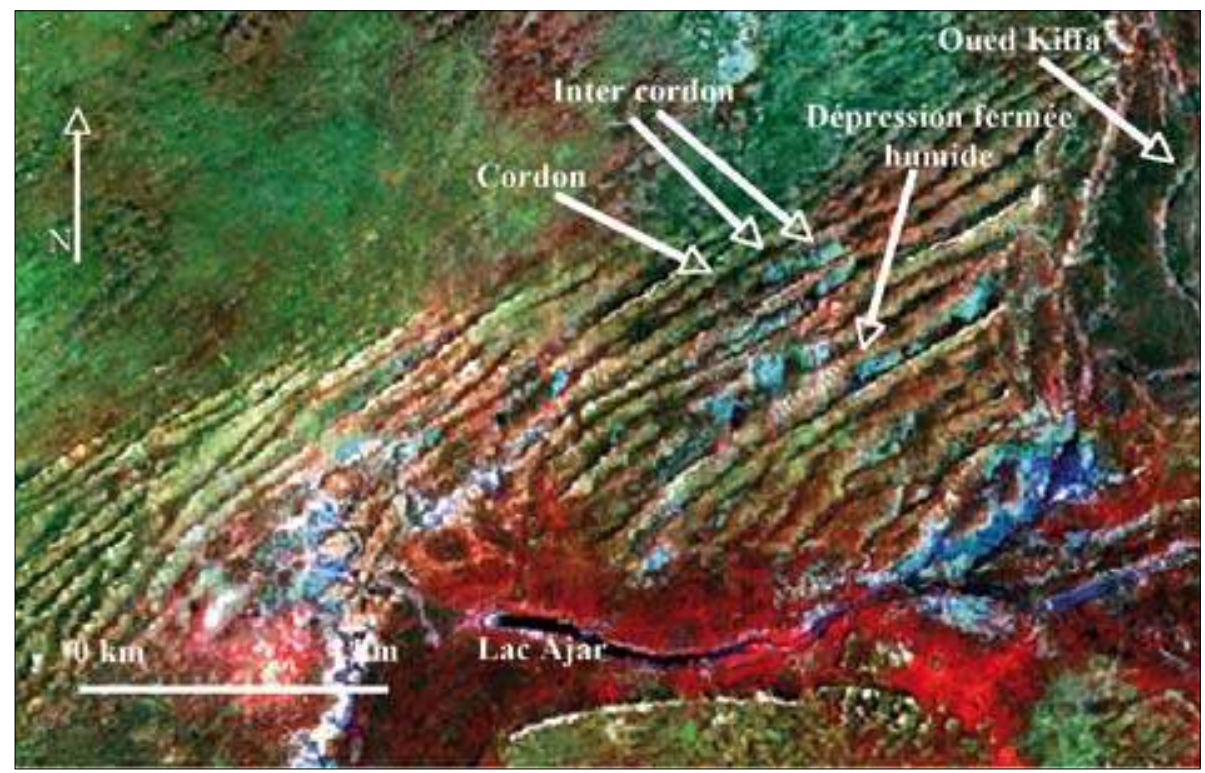

Figure 3 : Le système de cordons d'Ajar (Image Landsat TM, septembre 2001)

Composition colorée (canaux 2, 5 et 4) montrant l'alternance des cordons (teintes brun verdâtre) et les inter cordons rocheux (turquoise) avec des dépressions fermées à végétation dense (rouge) ou en eau (bleu).

Ajar dune ridges system (Landsat TM; September 2001)

Colored composition of bands 2, 5 and 4. Brown-greenish colours show ridges, turquoise and blue colours show rocky inter ridges, with densely vegetated closed depressions (in red) or water flooded closed depressions (in dark blue).

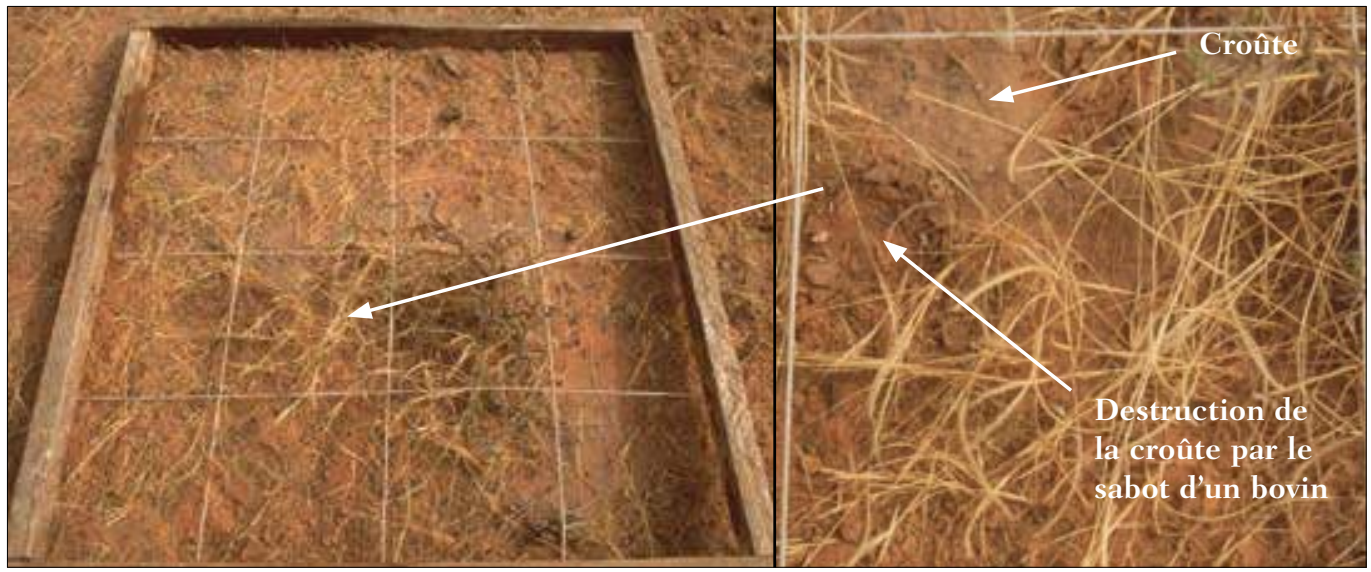

Photos 1 : Croûte de battance sur des sables faiblement limoneux (quadrat de $1 \times 1 \mathrm{~m}$ et carré de $0,25 \times 0,25 \mathrm{~m}$ ) Soil crust on lightly loamy sands ( 1 × 1 m quadrat and $0,25 \times 0,25 \mathrm{~m}$ quare) 
Planche VIII (Marie-Josée PENVEN et al. - Fonctionnement géomorphologique des régions...)

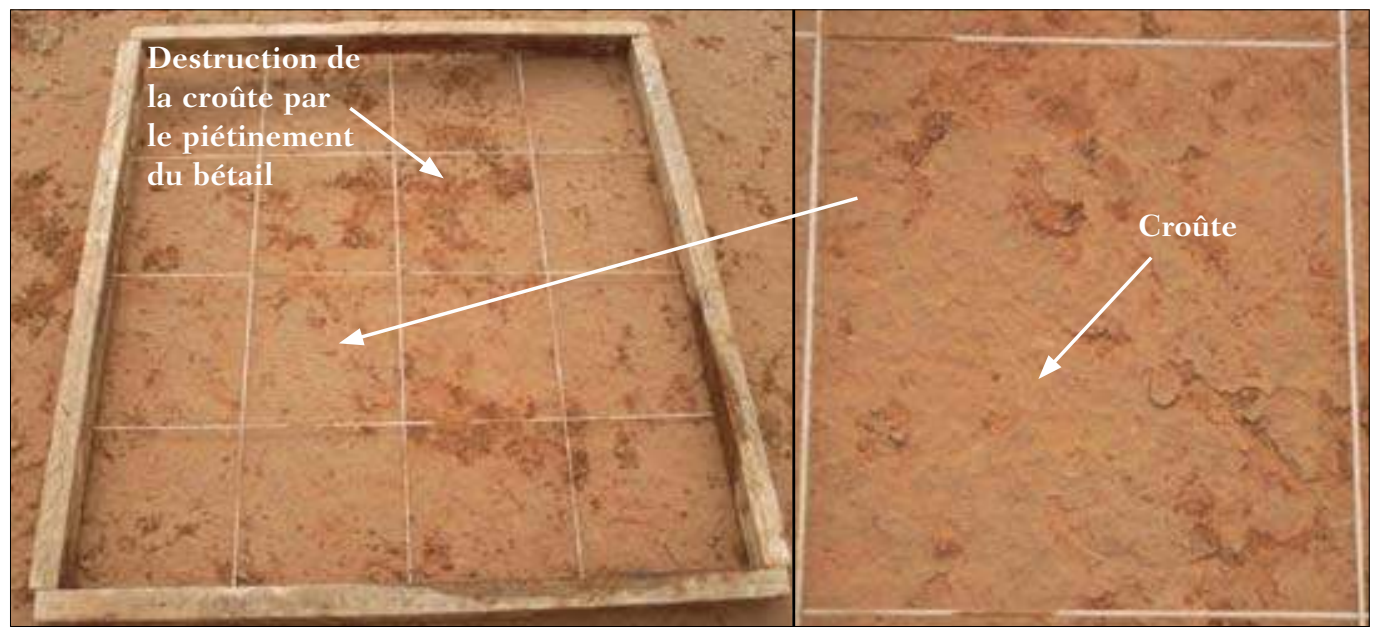

Photos 3 : Croûte de battance sur des sables rubéfiés

Le piétinement du bétail dégrade la croûte sans provoquer la dispersion des sables.

Soil crust on rubefied sands

Cattle stamp disturbs crust without sand dispersion

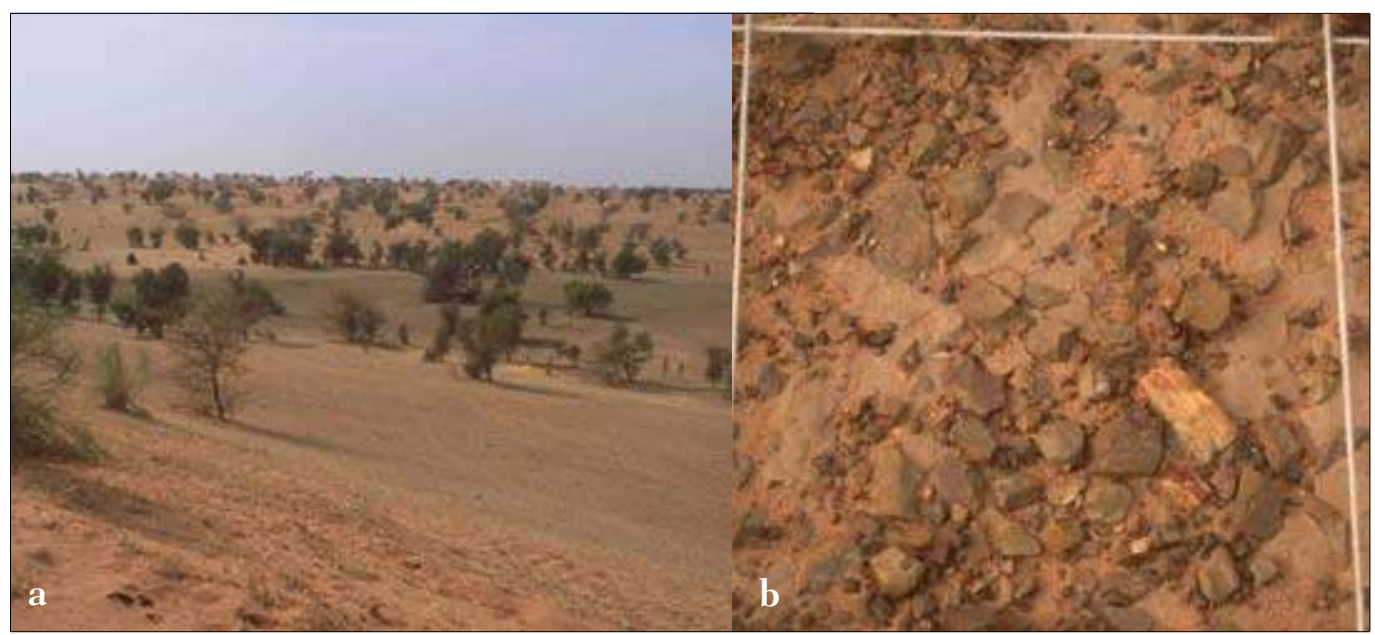

Photos 6 : Plages caillouteuses

a : reg en position intercordon; b : cailloutis sertis par des sables limoneux battants (carré du quadrat $0,25 \mathrm{x}$ $0,25 \mathrm{~m})$.

Gravel areas

a: a reg in an inter ridge; b: gravels set by loamy sands (square of quadrat: $0,25 \times 0,25 \mathrm{~m}$ ). 